\title{
A Julia-Carathéodory theorem for hyperbolically monotone mappings in the Hilbert ball
}

\author{
Mark Elin \\ Simeon Reich \\ and \\ David Shoikhet
}

\begin{abstract}
We establish a Julia-Carathéodory theorem and a boundary SchwarzWolff lemma for hyperbolically monotone mappings in the open unit ball of a complex Hilbert space.
\end{abstract}

Let $\mathbb{B}$ be the open unit ball of a complex Hilbert space $\mathcal{H}$ with inner product $\langle\cdot, \cdot\rangle$ and norm $\|\cdot\|$, and let $\rho: \mathbb{B} \times \mathbb{B} \mapsto \mathbb{R}^{+}$be the hyperbolic metric on $\mathbb{B}$ ([8], p. 98), i.e.,

$$
\rho(x, y)=\tanh ^{-1} \sqrt{1-\sigma(x, y)}
$$

where

$$
\sigma(x, y)=\frac{\left(1-\|x\|^{2}\right)\left(1-\|y\|^{2}\right)}{|1-\langle x, y\rangle|^{2}}, \quad x, y \in \mathbb{B} .
$$

We denote by $\mathcal{N}_{\rho}$ the class of all those self-mappings $F: \mathbb{B} \mapsto \mathbb{B}$ which are nonexpansive with respect to $\rho$ ( $\rho$-nonexpansive), i.e.,

$$
\rho(F(x), F(y)) \leq \rho(x, y) .
$$

Note that the class $\mathcal{N}_{\rho}$ properly contains the class $\operatorname{Hol}(\mathbb{B})$ of all holomorphic self-mappings of $\mathbb{B}([7,8])$.

Definition 1 A family $\mathcal{S}=\{F(t)\}_{t \geq 0}$ of self-mappings of $\mathbb{B}$ is said to be a one-parameter continuous semigroup (flow) on $\mathbb{B}$ if

$$
F(t+s)=F(t) \circ F(s), \quad t, s \geq 0,
$$

and

$$
\lim _{t \rightarrow 0^{+}} F(t)=I,
$$

where $I$ is the restriction of the identity mapping of $\mathcal{H}$ to $\mathbb{B}$ and the limit is taken pointwise with respect to the strong topology of $\mathcal{H}$. 
We denote $F(t) x$, the value of $F(t)$ at $x \in \mathbb{B}$, by $F_{t}(x), t \geq 0$.

Definition 2 A flow $\mathcal{S}=\{F(t)\}_{t \geq 0}$ on $\mathbb{B}$ is said to be generated if for each $x \in \mathbb{B}$, there exists the strong limit

$$
f(x):=\lim _{t \rightarrow 0^{+}} \frac{1}{t}\left(x-F_{t}(x)\right) .
$$
$\mathcal{S}$.

In this case the mapping $f: \mathbb{B} \mapsto \mathcal{H}$ is called the (infinitesimal) generator of

If $f$ generates a flow of $\rho$-nonexpansive self-mappings of $\mathbb{B}$, then we will write $f \in \mathcal{G N}_{\rho}(\mathbb{B})$.

The following result is established in [14]:

$\checkmark$ A semigroup $\mathcal{S}$ of holomorphic self-mappings of $\mathbb{B}$ is differentiable with respect to the parameter $t \geq 0$ (hence, generated by a holomorphic mapping) if and only if it is locally uniformly continuous on $\mathbb{B}$, i.e., the limit in Definition 1 is uniform on each $\rho$-ball in $\mathbb{B}$.

Moreover, in this case (see 9] and [15]):

- The generator $f$ is holomorphic on $\mathbb{B}$, and bounded and uniformly continuous on each $\rho$-ball in $\mathbb{B}$.

The set of all holomorphic semigroup generators is denoted by $\mathcal{G} \operatorname{Hol}(\mathbb{B})$.

The classical Julia-Carathéodory theorem and the boundary Schwarz-Wolff lemma play a crucial role in geometric function theory (see, for example, [3] and [19]). In particular, they can be effectively used in the study of the asymptotic behavior of discrete and continuous dynamical systems. In this context these celebrated results may be stated as follows:

- Let $F$ be a holomorphic self-mapping of the open unit disk $\Delta$ in the complex plane $\mathbb{C}$. If for a boundary fixed point $\tau \in \partial \Delta\left(\lim _{r \rightarrow 1^{-}} F(r \tau)=\tau\right)$ the angular derivative $\angle F^{\prime}(\tau):=\angle \lim _{z \rightarrow \tau} F^{\prime}(z)=k$ exists finitely, then

$$
\frac{|F(z)-\tau|^{2}}{1-|F(z)|^{2}} \leq k \frac{|z-\tau|^{2}}{1-|z|^{2}} .
$$

- If $k \leq 1$ this inequality means that each horocycle internally tangent to the unit circle $\partial \Delta$ at $\tau \in \partial \Delta$ is $F$-invariant.

- This is indeed the case when $F$ has no fixed point in $\Delta$ and $\tau \in \partial \Delta$ is its so-called Denjoy-Wolff point, that is, $\tau$ is an attractive fixed point for all orbits of $F$.

We use the symbol $\angle \lim _{z \rightarrow \tau}$ to denote the limit in each non-tangential approach region (see, for example, [12] and [19]).

Sometimes the above statements are grouped together under the name the Julia-Wolff-Carathéodory theorem. Higher dimensional analogs can be found, for instance, in 18, 8, 4, 3]. 
For holomorphic mappings on the open unit disk $\Delta$ in the complex plane $\mathbb{C}$ (i.e., for the one dimensional case when $\mathbb{B}=\Delta$ ), an infinitesimal version of the Julia-Wolff-Carathéodory theorem was given in [6]. Namely, it was shown there that

$\checkmark f \in \mathcal{G} \operatorname{Hol}(\Delta)$ has no null point in $\Delta$ if and only if for some $\tau \in \partial \Delta$, the angular derivative

$$
\angle f^{\prime}(\tau):=\angle \lim _{z \rightarrow \tau} f^{\prime}(z)=\alpha
$$

exists (finitely) with $\operatorname{Re} \alpha \geq 0$.

Moreover, $\alpha$ is, in fact, real and if $\mathcal{S}=\{F(t)\}_{t \geq 0}$ is the semigroup generated by $f$, then

$$
\frac{\left|F_{t}(z)-\tau\right|^{2}}{1-\left|F_{t}(z)\right|^{2}} \leq \exp (-t \alpha) \frac{|z-\tau|^{2}}{1-|z|^{2}}, \quad z \in \Delta, t \geq 0 .
$$

The point $\tau$ is unique and a (globally) attractive sink point of $\mathcal{S}$.

It is worth mentioning that the original Julia-Carathéodory theorem deals not only with attractive boundary fixed points, but also with repelling fixed points (see 3 16]), i.e., it deals with not necessarily fixed point free holomorphic self-mappings. In this direction, a generalization of the above theorem has recently been given by M. D. Contreras, S. Díaz-Madrigal and Ch. Pommerenke [2]. They proved the following one-dimensional assertion:

$\checkmark$ Let $f \in \mathcal{G} \operatorname{Hol}(\Delta)$. For a boundary point $\tau \in \partial \Delta$, the following claims are equivalent:

(i) the angular limit $\angle f^{\prime}(\tau):=\angle \lim _{z \rightarrow \tau} \frac{f(z)}{z-\tau}=\beta$ exists finitely;

(ii) the angular limit $\frac{d F_{t}(\tau)}{d z}:=\angle \lim _{z \rightarrow \tau} \frac{F_{t}(z)-\tau}{z-\tau}=\exp (-t \beta)$.

Note that condition (ii) is equivalent to inequality (8) for some $\alpha \leq \beta$.

In this paper we will establish these assertions for a general complex Hilbert (not necessarily finite-dimensional) space $\mathcal{H}$. Moreover, we will show that replacing the angular derivatives by just radial derivatives, we are able to prove infinitesimal versions of the Julia-Carathéodory theorem and the boundary Schwarz-Wolff lemma for the much wider class of generators of semigroups of $\rho$-nonexpansive self-mappings of the Hilbert ball $\mathbb{B}$. For the case of the Denjoy-Wolff point, the asymptotic behavior of one-parameter semigroups of $\rho$-nonexpansive and holomorphic mappings was also studied in [5].

Indeed, the content of the classical Schwarz-Pick lemma is the fact that each holomorphic self-mapping of the open unit disk in the complex plane is nonexpansive with respect to the Poincaré hyperbolic metric $\rho$ defined by (11). Therefore, one can try to use metric fixed point theory to derive results regarding those mappings which are nonexpansive with respect to $\rho$. At the same time, we must remember that if a given mapping (or semigroup) is not holomorphic, then the notion of derivative makes no sense.

It turns out, however, that although the study of the asymptotic behavior of a fixed point free semigroup consisting of $\rho$-nonexpansive mappings is, in 
general, much more complicated, one can define the real part of the radial derivative of its generator at a boundary fixed point and use it to find invariant ellipsoids, internally tangent at this point to the unit sphere, in the spirit of the Julia-Carathéodory theorem and the boundary Schwarz-Wolff lemma. To understand this phenomenon, we first consider the following example.

Example 1. Consider the continuous function $f: \bar{\Delta} \mapsto \mathbb{C}$ defined by

$$
f(z)=\frac{z+\bar{z}}{2}+\chi \frac{z-\bar{z}}{2}-1,
$$

where $\chi$ is a real parameter. Elementary calculations show that for all $\chi \geq \frac{1}{2}$ the following boundary flow invariance condition holds:

$$
\operatorname{Re} f(z) \bar{z} \geq 0, \quad z \in \partial \Delta .
$$

Therefore it follows from Martin's theorem [11] (see also [13) that $f$ generates a semigroup of continuous self-mappings of $\bar{\Delta}$. Indeed, solving the Cauchy problem

$$
\left\{\begin{array}{l}
\frac{\partial F_{t}(z)}{\partial t}+f\left(F_{t}(z)\right)=0, \\
F_{0}(z)=z, \quad z \in \Delta,
\end{array}\right.
$$

we find

$$
F_{t}(z)=1-e^{-t}+e^{-t} \frac{z+\bar{z}}{2}+e^{-\chi t} \frac{z-\bar{z}}{2}, \quad z \in \bar{\Delta} .
$$

It is clear that $F_{t}(1)=1$ for all $t \geq 0$ and $\lim _{t \rightarrow \infty} F_{t}(z)=1$ for all $z \in \bar{\Delta}$.

However, no more information on invariant sets of this semigroup can be obtained in this way.

If $\chi=1$, then for each $t \geq 0$, the function $F_{t}(z)$, as well as $f$, are holomorphic. Hence one can apply the result in [6] (see (77)-(8) above) to derive

$$
d_{1}\left(F_{t}(z)\right):=\frac{\left|1-F_{t}(z)\right|^{2}}{1-|z|^{2}} \leq \frac{e^{-t}|1-z|^{2}}{1-|z|^{2}}
$$

because the angular derivative of $f$ at the boundary fixed point $\tau=1$ exists and equals 1 .

But for $\chi>1$ our generator $f$, as well as its generated semigroup, are again not holomorphic.

However, fortunately, in this situation $(\chi>1)$ one can show that the semigroup $\mathcal{S}=\left\{F_{t}\right\}$ consists of $\rho$-nonexpansive self-mappings of the open unit disk $\Delta$ with the Poincaré hyperbolic metric $\rho$ defined on it. Indeed,

$$
F_{t}(z)=\left(1-e^{-t}\right)+z\left(\frac{e^{-t}}{2}+\frac{e^{-\chi t}}{2}\right)+\bar{z}\left(\frac{e^{-t}}{2}-\frac{e^{-\chi t}}{2}\right)
$$

is a convex combination of holomorphic and anti-holomorphic (hence, $\rho$-nonexpansive) mappings. So, by Theorem 6.5 on page 75 of $\left[\underline{8}, F_{t}\right.$ also is $\rho$-nonexpansive. 
In addition, we see that although $f$ is not differentiable in the complex sense in $\Delta$, its radial derivative at the boundary null point $\tau=1$ does exist:

$$
\alpha:=\lim _{r \rightarrow 1^{-}} \frac{f(r)}{r-1}=1 .
$$

So, the following question arises:

- Is this fact sufficient to ensure the same invariance condition as (10) for a semigroup of $\rho$-nonexpansive self-mappings of the open unit disk $\Delta$ ?

In this paper we give an affirmative answer to this question in a much more general situation.

To formulate our results, we need the following notions and notations.

For a fixed $\tau \in \partial \mathbb{B}$, the boundary of $\mathbb{B}$, and an arbitrary $x \in \mathbb{B}$, we define a non-Euclidean "distance" between $x$ and $\tau$ by the formula

$$
d_{\tau}(x)=\frac{|1-\langle x, \tau\rangle|^{2}}{1-\|x\|^{2}} .
$$

The sets

$$
E(\tau, s)=\left\{x \in \mathbb{B}: d_{\tau}(x)=\frac{|1-\langle x, \tau\rangle|^{2}}{1-\|x\|^{2}}<s\right\}, \quad s>0,
$$

are ellipsoids internally tangent to the unit sphere $\partial \mathbb{B}$ at $\tau$.

As in 1, it can be shown that the support functional $x^{*}$ of the smooth convex set $E(\tau, s)$ at $x \in \partial E(\tau, s), x \neq \tau$, normalized by the condition

$$
\lim _{x \rightarrow \tau}\left\langle x-\tau, x^{*}\right\rangle=1,
$$

can be expressed by

$$
x^{*}=\frac{1}{1-\|x\|^{2}} x-\frac{1}{1-\langle\tau, x\rangle} \tau .
$$

Theorem. Let $\mathcal{S}$ be a semigroup of $\rho$-nonexpansive self-mappings of the Hilbert ball $\mathbb{B}$, generated by $f: \mathbb{B} \mapsto \mathcal{H}$. Suppose that $f$ is uniformly continuous on each $\rho$-ball in $\mathbb{B}$, and $\tau \in \partial \mathbb{B}$ is a null point of $f$ in the sense that $\lim _{r \rightarrow 1^{-}} f(r \tau)=0$. The following assertions are equivalent:

$$
\limsup _{r \rightarrow 1^{-}} \operatorname{Re} \frac{\langle f(r \tau), \tau\rangle}{r-1}>-\infty
$$

(II) $\alpha:=\lim _{r \rightarrow 1^{-}} \operatorname{Re} \frac{\langle f(r \tau), \tau\rangle}{r-1}$ exists finitely;

(III) $\quad \beta:=\inf 2 \operatorname{Re}\left\langle f(x), x^{*}\right\rangle>-\infty$; 
(IV) there exists a real number $\gamma$ such that

$$
d_{\tau}\left(F_{t}(x)\right) \leq \exp (-t \gamma) \cdot d_{\tau}(x), \quad x \in \mathbb{B} .
$$

Moreover,

(a) $\alpha=\beta$ and the maximal $\gamma$ for which (IV) holds is exactly the same $\beta$;

(b) if $f$ is holomorphic and one (hence all) of conditions (I)-(IV) holds, then

$$
\lim _{r \rightarrow 1^{-}} \frac{\langle f(r \tau), \tau\rangle}{r-1}
$$

exists and is actually a real number.

Combining this result with Theorem 8.3 in [17], we arrive at the following analog of the boundary Schwarz-Wolff lemma.

Corollary. Let $\mathcal{S}=\left\{F_{t}\right\}_{t \geq 0} \subset \mathcal{N}_{\rho}$ be a semigroup of $\rho$-nonexpansive selfmappings of $\mathbb{B}$ generated by $f \in \mathcal{G N} \mathcal{N}_{\rho}(\mathbb{B})$. Assume that $f$ is null point free (that is, $\mathcal{S}$ has no stationary point in $\mathbb{B})$. Then there is a unique point $\tau \in \partial \mathbb{B}$ such that

$$
d_{\tau}\left(F_{t}(x)\right) \leq \exp (-t \alpha) \cdot d_{\tau}(x), \quad x \in \mathbb{B}
$$

for some $\alpha \geq 0$, and there is a continuous curve $\{x(r): 0 \leq r<1\} \subset \mathbb{B}$ ending at $\tau \in \partial \mathbb{B}$ for which

$$
\lim _{r \rightarrow 1^{-}} f(x(r))=0 .
$$

Conversely, if for some point $\tau \in \partial \mathbb{B}$ the radial limit $\lim _{r \rightarrow 1^{-}} f(r \tau)=0$ and the radial limit $\alpha:=\lim _{r \rightarrow 1^{-}} \operatorname{Re} \frac{\langle f(r \tau), \tau\rangle}{r-1}$ exists and is positive, then for each $t>0$, the mapping $F_{t}$ has no fixed point in $\mathbb{B}$.

To prove our theorem we need the following additional concepts and facts.

A mapping $f: \mathbb{B} \mapsto \mathcal{H}$ is said to be hyperbolically monotone or $\rho$-monotone (15, 10]) if for each pair $x, y \in \mathbb{B}$,

$$
\rho(x+r f(x), y+r f(y)) \geq \rho(x, y)
$$

for all $r \geq 0$ such that the points $x+r f(x)$ and $y+r f(y)$ belong to $\mathbb{B}$.

The crucial point in our approach is the following characterization of $\rho$ monotonicity [15, Theorem 2.1]:

$\checkmark$ A mapping $f: \mathbb{B} \mapsto \mathcal{H}$ is $\rho$-monotone if and only if

$$
\operatorname{Re}\left[\frac{\langle f(x), x\rangle}{1-\|x\|^{2}}+\frac{\langle y, f(y)\rangle}{\left.1-\|y\|^{2}\right)}\right] \geq \operatorname{Re}\left[\frac{\langle f(x), y\rangle+\langle x, f(y)\rangle}{1-\langle x, y\rangle}\right]
$$

for all points $x, y \in \mathbb{B}$.

Moreover, if $f \in \mathcal{G N}_{\rho}(\mathbb{B})$ is uniformly continuous on each $\rho$-ball in $\mathbb{B}$, then it is $\rho$-monotone. 
So, each holomorphic generator on $\mathbb{B}$ is $\rho$-monotone.

Proof of Theorem. The implication (II) $\Rightarrow$ (I) is trivial. To prove other implications we denote by $\psi$ the following real-valued function:

$$
\psi(t, x):=d_{\tau}\left(F_{t}(x)\right) .
$$

By direct calculation we get

$$
\left.\frac{\partial \psi(t, x)}{\partial t}\right|_{t=0}=-2 \psi(0, x) \operatorname{Re}\left\langle f(x), x^{*}\right\rangle,
$$

where $x^{*}$ is defined by (13). Hence, by the semigroup property,

$$
\frac{\partial \psi(t, x)}{\partial t}=-2 \psi(t, x) \operatorname{Re}\left\langle f\left(F_{t}(x)\right),\left(F_{t}(x)\right)^{*}\right\rangle
$$

for all $t \geq 0$.

Step 1. (I) $\Rightarrow$ (III). If (I) holds, then there exists a sequence $\left\{r_{n}\right\}$ such that $r_{n} \nearrow 1$ and

$$
\alpha_{1}:=\limsup _{r \rightarrow 1^{-}} \operatorname{Re} \frac{\langle f(r \tau), \tau\rangle}{r-1}=\lim _{n \rightarrow \infty} \operatorname{Re} \frac{\left\langle f\left(r_{n} \tau\right), \tau\right\rangle}{r_{n}-1} .
$$

Setting $y=r_{n} \tau$ in (17), we get

$$
\operatorname{Re} \frac{\langle f(x), x\rangle}{1-\|x\|^{2}}+\frac{\left\langle f\left(r_{n} \tau\right), r_{n} \tau\right\rangle}{1-r_{n}^{2}} \geq \operatorname{Re} \frac{\left\langle f(x), r_{n} \tau\right\rangle+\left\langle x, f\left(r_{n} \tau\right)\right\rangle}{1-\left\langle x, r_{n} \tau\right\rangle}
$$

or, equivalently,

$$
\begin{array}{r}
\operatorname{Re}\left\langle f(x), \frac{x}{1-\|x\|^{2}}-\frac{r_{n} \tau}{1-r_{n}\langle\tau, x\rangle}\right\rangle \geq \\
\quad \operatorname{Re}\left\langle f\left(r_{n} \tau\right), \frac{x}{1-r_{n}\langle x, \tau\rangle}-\frac{r_{n} \tau}{1-r_{n}^{2}}\right\rangle .
\end{array}
$$

Letting now $n$ tend to $\infty$, we see that

$$
\operatorname{Re}\left\langle f(x), x^{*}\right\rangle \geq \frac{\alpha_{1}}{2},
$$

i.e., (III) holds and $\beta$ is not less than $\alpha_{1}$. By the way, this implies that $\alpha_{1}$ is finite.

Step 2. (III) $\Leftrightarrow(I V)$. Let (IV) hold for some real number $\gamma$. Differentiating (15) at $t=0$, we obtain

$$
2 \operatorname{Re}\left\langle f(x), x^{*}\right\rangle \geq \gamma
$$

i.e., (III) holds, and $\beta \geq \gamma$.

Let now (III) hold. Then by (19),

$$
\frac{\partial \psi(t, x)}{\partial t} \leq-\psi(t, x) \beta
$$


Integrating this inequality with respect to $t$, we see that (IV) holds with $\gamma=\beta$.

Step 3. (III) $\Rightarrow(\mathrm{I})$. Let

$$
2 \operatorname{Re}\left\langle f(x), \frac{x}{1-\|x\|^{2}}-\frac{\tau}{1-\langle\tau, x\rangle}\right\rangle \geq \beta .
$$

Substituting $x=r \tau, 0<r<1$, we see that

$$
2 \operatorname{Re}\left\langle f(r \tau), \frac{r \tau}{1-r^{2}}-\frac{\tau}{1-r}\right\rangle \geq \beta
$$

or

$$
\operatorname{Re} \frac{\langle f(r \tau), \tau\rangle}{r-1} \cdot \frac{2}{r+1} \geq \beta .
$$

Therefore

$$
\liminf _{r \rightarrow 1^{-}} \operatorname{Re} \frac{\langle f(r \tau), \tau\rangle}{r-1} \geq \beta
$$

Hence (I) holds and $\limsup _{r \rightarrow 1^{-}} \operatorname{Re} \frac{\langle f(r \tau), \tau\rangle}{r-1} \geq \beta$.

Step 4. Just by comparing Step 1 and Step 3, we conclude that (III) implies (II), i.e., if (III) holds, then $\alpha=\lim _{r \rightarrow 1^{-}} \operatorname{Re} \frac{\langle f(r \tau), \tau\rangle}{r-1}$ exists and is equal to $\beta$.

Step 5. To end the proof, we have to prove (b).

Suppose that $f$ is holomorphic. We introduce a holomorphic function $g \in$ $\operatorname{Hol}(\Delta, \mathbb{C})$ as follows:

$$
g(\lambda):=\langle f(\lambda \tau), \tau\rangle-\frac{\beta}{2}\left(\lambda^{2}-1\right) .
$$

It follows from (III) that

$$
\operatorname{Re}\left\langle f(\lambda \tau),(\lambda \tau)^{*}\right\rangle \geq \frac{\beta}{2}
$$

On the other hand,

$$
\begin{aligned}
& \left\langle f(\lambda \tau),(\lambda \tau)^{*}\right\rangle=\left\langle f(\lambda \tau), \frac{\lambda \tau}{1-|\lambda|^{2}}-\frac{\tau}{1-\bar{\lambda}}\right\rangle \\
& =\langle f(\lambda \tau), \tau\rangle \cdot\left(\frac{\bar{\lambda}}{1-|\lambda|^{2}}-\frac{1}{1-\lambda}\right) \\
& =\left(g(\lambda)+\frac{\beta}{2}\left(\lambda^{2}-1\right)\right) \cdot \frac{\bar{\lambda}-1}{\left(1-|\lambda|^{2}\right)(1-\lambda)} \\
& =g(\lambda) \cdot \frac{|\lambda-1|^{2}}{-\left(1-|\lambda|^{2}\right)(1-\lambda)^{2}}+\frac{\beta}{2} \frac{(1-\bar{\lambda})(1+\lambda)}{1-|\lambda|^{2}} .
\end{aligned}
$$

Therefore $\operatorname{Re} \frac{g(\lambda)}{-(1-\lambda)^{2}} \geq 0$. By the Riesz-Herglotz representation formula,

$$
\frac{g(\lambda)}{-(1-\lambda)^{2}}=\oint_{|\zeta|=1} \frac{1+\lambda \bar{\zeta}}{1-\lambda \bar{\zeta}} d \sigma(\zeta)
$$


where $d \sigma(\zeta)$ is a positive measure on the unit circle. Decomposing $d \sigma$ with respect to Dirac's $\delta$-function at the point $\zeta=1, d \sigma(\zeta)=a \delta(\zeta)+d \sigma_{1}(\zeta), a \geq 0$, we calculate:

$$
\lim _{r \rightarrow 1^{-}} \frac{g(r)}{r-1}=\lim _{r \rightarrow 1^{-}}(1-r) \oint_{|\zeta|=1} \frac{1+r \bar{\zeta}}{1-r \bar{\zeta}}\left(a \delta(\zeta)+d \sigma_{1}(\zeta)\right)=2 a \geq 0 .
$$

This fact, in turn, implies that

$$
\lim _{r \rightarrow 1^{-}} \frac{\langle f(r \tau), \tau\rangle}{r-1}=\lim _{r \rightarrow 1^{-}}\left(\frac{g(r)}{r-1}+\frac{\beta}{2}(r+1)\right)=2 a+\beta
$$

exists and is real.

Since by Step $4, \operatorname{Re}(2 a+\beta)=\beta$, we have that $a=0$, and hence the limit

$$
\lim _{r \rightarrow 1^{-}} \frac{\langle f(r \tau), \tau\rangle}{r-1}=\beta
$$

is real. This completes the proof of our theorem.

Example 2. Let $\mathcal{H}_{1}$ be a Hilbert space. Consider the semigroup of holomorphic self-mappings of the unit ball $\mathbb{B}$ of the space $\mathcal{H}:=\mathbb{C} \times \mathcal{H}_{1}$ defined by the formula

$$
F_{t}\left(z_{1}, z_{2}\right)=\left(\frac{z_{1}}{z_{1}+e^{t}\left(1-z_{1}\right)}, \frac{e^{t / 2} z_{2}}{z_{1}+e^{t}\left(1-z_{1}\right)}\right),
$$

where $z_{1} \in \mathbb{C}, z_{2} \in \mathcal{H}_{1},\left|z_{1}\right|^{2}+\left\|z_{2}\right\|^{2}<1$. This semigroup has a boundary fixed point $\tau=(1,0)$ which is not its Denjoy-Wolff point. Consider the semigroup generator $f \in \operatorname{Hol}(\mathbb{B}, \mathcal{H})$ :

$$
f\left(z_{1}, z_{2}\right)=-\left.\frac{\partial F_{t}\left(z_{1}, z_{2}\right)}{\partial t}\right|_{t=0}=\left(z_{1}\left(1-z_{1}\right), \frac{\left(1-2 z_{1}\right) z_{2}}{2}\right) .
$$

Now we just calculate

$$
\alpha=\lim _{r \rightarrow 1^{-}} \frac{\langle f(r \tau), \tau\rangle}{r-1}=-1 .
$$

Hence,

$$
d_{\tau}\left(F_{t}(z)\right) \leq e^{t} d_{\tau}(z), \quad z \in \mathbb{B}, t \geq 0 .
$$

Example 3. Define another semigroup $\left\{F_{t}\right\}_{t \geq 0}=\left\{\left(F_{t}\right)_{1},\left(F_{t}\right)_{2}\right\}_{t \geq 0}$ on the unit ball $\mathbb{B}$ of the same space $\mathcal{H}$ by the formulae:

$$
\begin{aligned}
& \left(F_{t}\right)_{1}\left(z_{1}, z_{2}\right)=\frac{\left(1+z_{1}^{2}\right) e^{2 t}-\left(1-z_{1}\right) \sqrt{2\left(1+z_{1}^{2}\right) e^{2 t}-\left(1-z_{1}\right)^{2}}}{\left(1+z_{1}^{2}\right) e^{2 t}-\left(1-z_{1}\right)^{2}}, \\
& \left(F_{t}\right)_{2}\left(z_{1}, z_{2}\right)=z_{2} \sqrt{\frac{\partial\left(F_{t}\right)_{1}\left(z_{1}, z_{2}\right)}{\partial z_{1}}}
\end{aligned}
$$


where $z_{1} \in \mathbb{C}, z_{2} \in \mathcal{H}_{1},\left|z_{1}\right|^{2}+\left\|z_{2}\right\|^{2}<1$. Consider its generator $f \in \operatorname{Hol}(\mathbb{B}, \mathcal{H})$ :

$f\left(z_{1}, z_{2}\right)=-\left.\frac{\partial F_{t}\left(z_{1}, z_{2}\right)}{\partial t}\right|_{t=0}=\left(-\frac{\left(1-z_{1}\right)\left(1+z_{1}^{2}\right)}{1+z_{1}}, \frac{z_{2}\left(1-z_{1}+z_{1}^{2}+z_{1}^{3}\right)}{\left(1+z_{1}\right)^{2}}\right)$.

It is clear that $f$ has three boundary null points: $\tau_{1}=(1,0), \tau_{2}=(i, 0)$ and $\tau_{3}=(-i, 0)$. For each one of them we just calculate

$$
\begin{aligned}
& \alpha_{1}=\lim _{r \rightarrow 1^{-}} \frac{\left\langle f\left(r \tau_{1}\right), \tau_{1}\right\rangle}{r-1}=\lim _{r \rightarrow 1^{-}} \frac{-(1-r)\left(1+r^{2}\right)}{(1+r)(r-1)}=1, \\
& \alpha_{2}=\lim _{r \rightarrow 1^{-}} \frac{\left\langle f\left(r \tau_{2}\right), \tau_{2}\right\rangle}{r-1}=\lim _{r \rightarrow 1^{-}} \frac{-(1-r i)\left(1-r^{2}\right)(-i)}{(1+r i)(r-1)}=-2, \\
& \alpha_{3}=\lim _{r \rightarrow 1^{-}} \frac{\left\langle f\left(r \tau_{3}\right), \tau_{3}\right\rangle}{r-1}=\lim _{r \rightarrow 1^{-}} \frac{-(1+r i)\left(1-r^{2}\right) i}{(1-r i)(r-1)}=-2 .
\end{aligned}
$$

Hence, the following three inequalities hold simultaneously for all $z \in \mathbb{B}$ and $t \geq 0$ :

$$
\begin{gathered}
d_{\tau_{1}}\left(F_{t}(z)\right) \leq e^{-t} d_{\tau_{1}}(z), \\
d_{\tau_{2}}\left(F_{t}(z)\right) \leq e^{2 t} d_{\tau_{2}}(z), \\
d_{\tau_{3}}\left(F_{t}(z)\right) \leq e^{2 t} d_{\tau_{3}}(z) .
\end{gathered}
$$

These inequalities mean that for each point $z \in \mathbb{B}$ and for each $t \geq 0$, the image $F_{t}(z)$ belongs to the intersection of the ellipsoids:

$$
E\left(\tau_{1}, e^{-t} d_{\tau_{1}}(z)\right) \bigcap E\left(\tau_{2}, e^{2 t} d_{\tau_{2}}(z)\right) \bigcap E\left(\tau_{3}, e^{2 t} d_{\tau_{3}}(z)\right) .
$$

Example 4. Consider the one-parameter continuous semigroup $\mathcal{S}=\left\{F_{t}\right\}_{t \geq 0}$, consisting of holomorphic self-mappings of the open unit disk $\Delta$ in the complex plane, defined by

$$
F_{t}(z)=1-(1-\exp (-t)+\exp (-t) \sqrt{1-z})^{2}, \quad z \in \Delta, t \geq 0 .
$$

One can check that $\mathcal{S}$ is generated by the following function:

$$
f(z)=-2 \sqrt{1-z}(\sqrt{1-z}-1) .
$$

It is easy to see that $f(1)=0$, but $f$ has no angular derivative at the point $z=1$. At the same time, this point is not even a fixed point of $\mathcal{S}$.

Example 5. Now we consider the semigroup $\mathcal{S}=\left\{F_{t}\right\}_{t \geq 0} \subset \operatorname{Hol}(\Delta)$ defined by

$$
F_{t}(z)=\frac{(1+z)^{\alpha(t)}-(1-z)^{\alpha(t)}}{(1+z)^{\alpha(t)}+(1-z)^{\alpha(t)}}
$$

where $\alpha(t)=e^{-2 t}$. Differentiating at $t=0$, we find its generator:

$$
f(z)=\left(1-z^{2}\right) \log \frac{1+z}{1-z} .
$$


Similarly as in the previous example, $f( \pm 1)=0$, but $f$ has no angular derivative at the points $\tau_{1}=1$ and $\tau_{2}=-1$. However, in contrast with that example, these points are fixed points of the semigroup:

$$
F_{t}( \pm 1)= \pm 1 \text { for all } t \geq 0 .
$$

Moreover, it is possible to calculate the "non-Euclidean distance" $d_{ \pm 1}\left(F_{t}(z)\right)$. In particular, for real $z=x$ we have:

$$
d_{1}\left(F_{t}(x)\right)=\frac{1-F_{t}(x)}{1+F_{t}(x)}=\left(\frac{1-x}{1+x}\right)^{\alpha(t)}=d_{1}(x)^{\alpha(t)} .
$$

Since $0<\alpha(t)<1$ when $t>0$, we conclude that for each fixed $t>0$, the quotient $\frac{d_{1}\left(F_{t}(x)\right)}{d_{1}(x)}$ tends to infinity as $z=x$ tends to 1 radially, i. e., $d_{1}\left(F_{t}(z)\right)$ does not admit an estimate of the form $A(t) d_{1}(z)$.

Acknowledgment. The second author was partially supported by the Fund for the Promotion of Research at the Technion and by the Technion VPR Fund - B. and G. Greenberg Research Fund (Ottawa).

\section{References}

[1] D. Aharonov, M. Elin, S. Reich and D. Shoikhet, Parametric representations of semi-complete vector fields on the unit balls in $\mathbb{C}^{n}$ and in Hilbert space, Atti Accad. Naz. Lincei Cl. Sci. Fis. Mat. Natur. Rend. Lincei (9) Mat. Appl. 10 (1999), 229-253.

[2] M. D. Contreras, S. Díaz-Madrigal and Ch. Pommerenke, On boundary critical points for semigroups of analytic functions, Math. Scand. 98 (2006), $125-142$.

[3] C. C. Cowen and B. D. MacCluer, Composition Operators on Spaces of Analytic Functions, CRC Press, Boca Raton, FL, 1995.

[4] S. Dineen, The Schwarz Lemma, Clarendon Press, Oxford, 1989.

[5] M. Elin, S. Reich and D. Shoikhet, Asymptotic behavior of semigroups of $\rho$-nonexpansive and holomorphic mappings on the Hilbert ball, Ann. Mat. Pura Appl. (4) 181 (2002), 501-526.

[6] M. Elin and D. Shoikhet, Dynamic extension of the Julia-WolffCarathéodory theorem, Dynam. Systems Appl. 10 (2001), 421-438.

[7] T. Franzoni and E. Vesentini, Holomorphic Maps and Invariant Distances, North-Holland, Amsterdam, 1980. 
[8] K. Goebel and S. Reich, Uniform Convexity, Hyperbolic Geometry and Nonexpansive Mappings, Marcel Dekker, New York and Basel, 1984.

[9] L. A. Harris, S. Reich and D. Shoikhet, Dissipative holomorphic functions, Bloch radii, and the Schwarz lemma, J. Anal. Math. 82 (2000), 221-232.

[10] E. Kopecká and S. Reich, Hyperbolic monotonicity in the Hilbert ball, Fixed Point Theory Appl. 2006, Article ID 78104, 1-15.

[11] R. H. Martin, Jr., Differential equations on closed subsets of a Banach space, Trans. Amer. Math. Soc. 179 (1973), 399-414.

[12] Ch. Pommerenke, Boundary Behavior of Conformal Maps, Springer, Berlin, 1992.

[13] S. Reich, On fixed point theorems obtained from existence theorems for differential equations, J. Math. Anal. Appl. 54 (1976), 26-36.

[14] S. Reich and D. Shoikhet, Generation theory for semigroups of holomorphic mappings in Banach spaces, Abstr. Appl. Anal. 1 (1996), 1-44.

[15] S. Reich and D. Shoikhet, Semigroups and generators on convex domains with the hyperbolic metric, Atti. Accad. Naz. Lincei Cl. Sci. Fis. Mat. Natur. Rend. Lincei (9) Mat. Appl. 8 (1997), 231-250.

[16] S. Reich and D. Shoikhet, The Denjoy-Wolff theorem, Encyclopaedia of Mathematics, Supplement III, Kluwer Academic Publishers, Dordrecht, 2001, 121-123.

[17] S. Reich and D. Shoikhet, Nonlinear Semigroups, Fixed Points, and Geometry of Domains in Banach Spaces, Imperial College Press, London, 2005.

[18] W. Rudin, Function Theory on the Unit Ball in $\mathbb{C}^{n}$, Springer, Berlin, 1980.

[19] J. H. Shapiro, Composition Operators and Classical Function Theory, Springer, Berlin, 1993.

[20] D. Shoikhet, Semigroups in Geometrical Function Theory, Kluwer, Dordrecht, 2001.

Mark Elin

Department of Mathematics,

ORT Braude College,

21982 Karmiel, Israel

E-mail address: mark.elin@gmail.com 


\section{Simeon Reich}

Department of Mathematics,

The Technion - Israel Institute of Technology,

32000 Haifa, Israel

E-mail address: sreich@tx.technion.ac.il

David Shoikhet

Department of Mathematics,

ORT Braude College,

21982 Karmiel, Israel

E-mail address: davs27@netvision.net.il 